Laparoscopic repair for recurrent hernia after prosthesis surgery (35 cases)

J. Cady, J. Godfroy, O. Sibaud, C. Kron (1997) Chirurgie 122: 539-544

With author's permission

The authors report 35 cases of laparoscopic repair after prosthesis surgery using the classic open technique (19 cases) or laparoscopic technique (16 cases). Most difficulties were observed after intraperitoneal laparoscopy. Hernia repair was preferentially intrapreperitoneal (31 cases). Postoperative morbidity was low with two cases of serohematic effusion treated by puncture. Mean hospital stay was 2.5 days. Patients returned to normal activity within 7 days but work stoppage in active patients depended more on patient motivation than the initial surgical procedure. There were no reiterative recurrences although follow-up is too short (mean 21.2 months) for definitive results.

\title{
Erratum
}

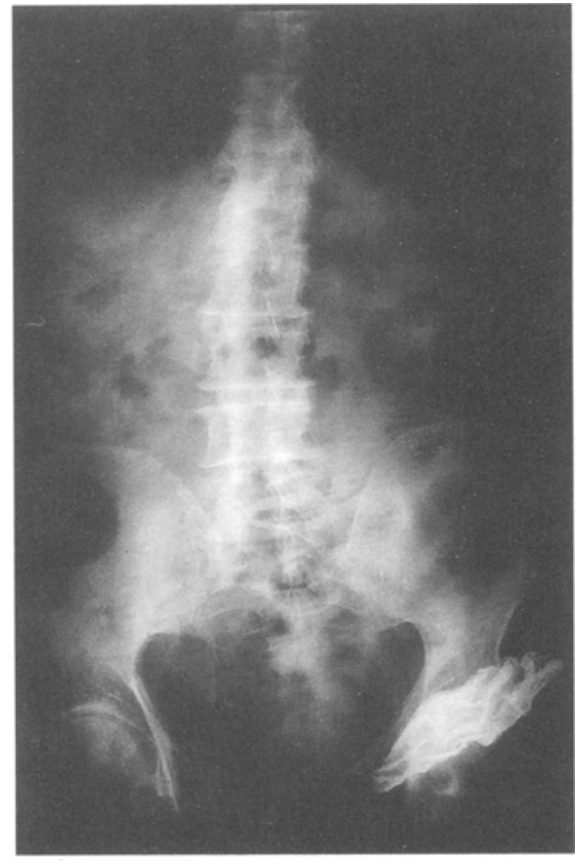

Fig. 1

Plain abdominal film showing metal mesh in left inguinal region
Hernia (1998) 2: 39-40

\section{Late colocutaneous fistula after mesh repair of an inguinal hernia}

\author{
S.H. Weitzel' ${ }^{1}$ A.J. Botha ${ }^{2}$ and P.A. Thomas ${ }^{2}$ \\ ${ }^{1}$ Department of Orthopaedics, Queen Mary's Hospital, Frognal Avenue, Sidcup DA14 6LT, UK \\ ${ }^{2}$ Department of Surgery, Whipps Cross Hospital, Whipps Cross Road, London E11 1NR, UK
}

Figure 2 has been wrongly duplicated and was reprinted instead of Figure 1. 